\title{
Merge Before Toll Based on the Maximum Traffic Flow
}

\author{
Feifei Han \\ Department of Economic Management, School of North China Electric Power University, Baoding \\ 071003, China \\ hff869803@163.com
}

Keyword: Toll station; The maximum traffic flow; Merging pattern; Dynamic programming

\begin{abstract}
Highway toll station is one of the most important components and is the only entrance of highway. The traffic capacity of highway is straightforward influenced by the capacity of toll station. So in order to acquire the optimal configuration of merging pattern before toll, this paper construct a model by the Dynamic Programming method. For maximize the throughput, the model designed the different merging pattern. Firstly, the model built the quadratic equation between the throughput and density. Secondly, vehicles are divided into three categories according to their types and speed: the fast vehicles, the medium speed vehicles and the slow vehicles. In accordance with speed, we divided the lines of toll station into the fast lines and the slow lines. The different merging pattern generated by the correspondence of vehicles and lines. Thirdly, we acquired the density when the throughput up to maximum. Finally, according to the density, determined the merging pattern. Meanwhile, in the merging pattern, the throughput up to maximum.
\end{abstract}

\section{Introduction}

Toll station on highway have strongly influence on highway operation and management. On the one hand, the amount of traffic accidents on toll station increasing continuously, for many reasons, mainly includes traffic participant, vehicles, toll station environment, management, weather conditions, and so on. On the other hand, the toll station is a basic unit of highway management facility and is distributed on borderland of city. There are more and more quantity of distribution point, which are expensive to construct.

The Reason for Toll Station Collects Tolls. At the present development stage of China, it is necessary to collect tolls from motorist after the highway was opened to the public, for the following reasons:

The highway is a commercial infrastructure. Because the funds on highway construction are from a variety of sources, which objectively determined the highway is a commercial property. It is necessary to return on construction capital, and that is why toll station collects tolls.

The highway is a kind of special merchandise. The highway has the higher differential benefit than ordinary road. The benefit mainly generated by the decrease of the transportation cost, the decline of the vehicles run time and accident. So the toll station collecting tolls from the vehicles. The tolls are used to compensate the costs of management and investment.

To meet the needs of control traffic. Toll collection can relieve the traffic congestion when the supply of road is inelastic.

Toll Collection Methods. As the reference material shows, there are a variety of methods to collect tolls on the highway, in China now. According to the automation degree of toll collection, the toll collection methods are divided into three types: Manual Toll collection (MTC)[3]: judged the information of vehicles via the staff and collect tolls.Manual Semi-automatic Toll collection: the management mode is to issue card in the entry line, read the ticket and collect toll in the exit lane.Electronic toll collection (ETC)[4]: RFID tags are used in toll collection system that let vehicles fly through toll stations without cash. Moreover, the ETC aims to eliminate the delay on toll roads by tolls electronically. 


\section{Assumptions}

The factors that we already have taken into consideration play a vital role in the model.

Cars have a wait time at the tollbooth, which can be ignored[5].

The toll plazas are not near on-ramps. We do not consider the possibility of additional cars merged only those that were already on the main road[6].

All lines of highways are equivalent to the same highways. We consider only divided lines.

The data that we have collected is enough and accurate and the quantification.

\section{Model: Determine Merging Pattern Via the Maximum Traffic}

\section{Symbols and Definitions}

Table 1 Symbols and definitions

\begin{tabular}{cc}
\hline Symbols & Definitions \\
\hline$q(t)$ & Traffic \\
$q_{i}(t)$ & The Traffic of Moment $t$ \\
$L$ & The Traffic of Moment $t$ and Line $i$ \\
& The Length of Road \\
$\rho_{j}$ & Vehicle Density \\
$\rho_{i}(t)$ & Jam Density \\
$v$ & The Vehicle Density of Moment $t$ and Line $i$ \\
$v_{i}(t)$ & Vehicle Speed \\
$v_{f}$ & The Vehicle Speed of Moment $t$ and Line $i$ \\
$\alpha, a, b, c, n_{1}, n_{2}, n_{3}$, & Cnrestricted Speed \\
\hline
\end{tabular}

\section{Construction}

Fundamental Formula. Firstly, the non-uniform traffic meeting the formula $q \mathrm{v}$. The ration between density and speed is $v=\alpha \rho+\beta$. Respectively, 0,0 , so the formula becomes $v=v_{f}\left(1-\frac{\rho}{\rho_{j}}\right)$

. And the relation between traffic and density is $q=v_{f}\left(\rho-\frac{\rho^{2}}{\rho_{j}}\right)$ relation between density, traffic and speed, we obtained the ration of traffic and speed. 


$$
q=v_{f}\left(\rho-\frac{v^{2}}{v^{f}}\right)
$$

Moreover, the optimal speed marked by $v_{m}$, so $v_{m}=\frac{v_{f}}{2}$.

Calculate Thought. The vehicles are divided into three types via its speed, includes fast vehicles (FV) whose maximum speed is $v_{1}$, medium-speed vehicles(MV) whose maximum speed is $v_{2}$, and low speed vehicles ( $\mathrm{LV}$ ) whose maximum speed is $\left.\begin{array}{llll}v_{3} & v_{1} & v_{2} & v_{3}\end{array}\right)$. The lanes are divided into two types, includes fast lines (FL) and low speed lines (LL). As FV $n_{2} \bullet L$, MV $n_{3} \bullet L$ and $\mathrm{LV}_{3} n_{3} L$ get through the toll station, it has a choice of two types lines (here, we assumed only two lines that one is FL, the other is LL.). And assumed that FV $a \cdot L, \mathrm{MV} b \cdot L$ and $\mathrm{LV} c \cdot L$ merge into the FL. The traffic of moment $t+1$ meet the formula,

$$
\begin{aligned}
& q(a, b, c)=\left[\rho_{1}(t)+a+b+c\right]\left[v_{1}\left(1-\frac{\rho_{1}(t)+a+b+c}{\rho_{j}}\right)\right]+\left[\rho_{2}(t)+n_{1}+n_{2}+n_{3}-a-b-c\right] \\
& {\left[v_{3}\left(1-\frac{\rho_{2}+n_{1}+n_{2}+n_{3}-a-b-c}{p_{j}}\right)\right]}
\end{aligned}
$$

There are several situations:

When $a=n_{1}, b=0$, the $c$ must be 0 . So the $q$ directly calculated.

When $a=n_{1}, b<n_{2}, c=0$, we can obtain the maximum traffic by differential. The maximum is corresponding to a value of $b$.

When $0<c<n_{3}$, it is certain that $a=n_{1}, b=n_{2}$. Then calculate the maximum of traffic.
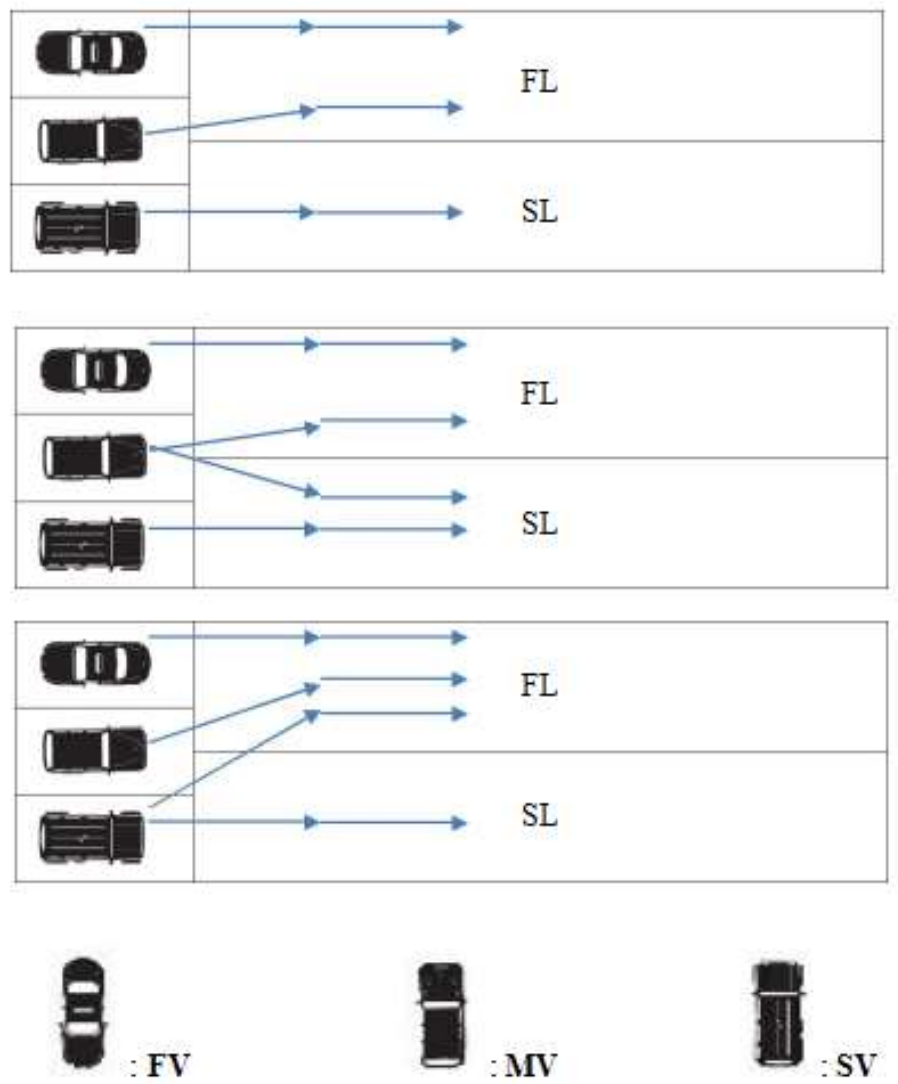

Figure 1. The merging pattern 
In conclusion, we use the same method to calculate the maximum of traffic in other situations.

\section{Conclusion}

The whole course of the method is that to take the derivative of the Formula 2 firstly.

Secondly, solve the unknown parameter ( $\mathrm{a}, \mathrm{b}$ or $\mathrm{c}$ ). Because the number of vehicles have already get through the tollbooth are certain. When the value of unknown parameter is certain, the quantity of merged into FL also are certain. Moreover, take the parameter into Formula 2, we can obtain the maximum of traffic. Finally, determine the merging pattern.

In spite of the quantity of line that can divide into several types, the different speed vehicles merge into the number of lines which also can use the method to acquire. In this case, the merging pattern also is certain.

\section{References}

[1] http://kns.cnki.net/KCMS/detail/detail. aspx?dbcode=CMFD\&dbname=CMFD9904\& filename $=2003114967$. nh\&uid $=$

[2] http://kns.cnki.net/kns/brief/default_result.aspx

[3] http://kns.cnki.net/KCMS/detail/detail.aspx?dbcode=CJFQ\&dbname=CJFD2000 \&filename $=$ HLJJ200006008\&uid=

[4] http://kns.cnki.net/KCMS/detail/detail.aspx $?$ dbcode=CMFD\&dbname=CMFD0506 \&filename $=2004133465$. nh\&uid $=$

[5] Robert R., Jr. 1963. Contribution of mathematical concepts to management. Industrial College of the Armed Forces http://www.ndu. edu/library/ic4/L64-015.pdf.

[6] Pratelli, Schoen. Optimal design of motorway toll stations[J].European Transport Conference,2003(9), 8:1-33 